\title{
Validade de Critério do Inventário de Potencial para Abuso Infantil (CAP)
}

\author{
Ana Carolina de Almeida Patrian \\ Karyne de Souza Augusto Rios \\ Lúcia Cavalcanti de Albuquerque Williams ${ }^{1}$ \\ Universidade Federal de São Carlos, São Carlos-SP, Brasil
}

\begin{abstract}
Resumo: É grande o interesse pelo tema da violência contra a criança, porém são escassas suas formas de avaliação, dificultando o desenvolvimento de intervenções. O Inventário de Potencial de Abuso Infantil (CAP) é considerado eficaz na identificação precoce de pais considerados em risco de agredirem fisicamente seus filhos e uma boa ferramenta para avaliar intervenções na área dos maus-tratos contra crianças e adolescentes. O presente estudo objetivou realizar a validade de critério do Inventário CAP, por meio da comparação de seus resultados entre cuidadores considerados de alto risco para abuso infantil e cuidadores de baixo risco para o abuso infantil. Dois grupos de pais foram participantes, sendo um grupo composto por 20 pais denunciados por agressões físicas e o outro grupo de 20 pais não agressores de igual nível socioeconômico e educacional. Os resultados indicaram que o inventário é capaz de avaliar as diferenças entre os grupos propostos, demonstrando ser um bom instrumento para o trabalho de serviços de proteção à criança epara a avaliação de intervenções e políticas públicas ou pesquisas.
\end{abstract}

Palavras-chave: abuso da criança, inventários, validade do teste, violência na família, avaliação psicológica

\section{Criterion Validity of the Child Abuse Potential Inventory (CAP)}

\begin{abstract}
Even though there is great interest in the field of child abuse, there are few methods to assess it, which hinders the development of interventions. The Child Abuse Potential (CAP) Inventory is considered effective in the early identification of parents at risk of physically abusing their children, and a good tool to evaluate interventions in the field of child abuse. This study's objective was to perform the CAP Inventory's criterion validity by comparing the results obtained by caregivers at a high risk of abusing their children with those obtained by caregivers at a low risk for child abuse. Two groups of parents participated in the study: a group of 20 parents reported for physically abusing their children and a group of 20 non-offending parents with the same socio-economic and educational levels. The results indicated the instrument is able to discriminate between the two groups, showing it to be a good tool for use in child protection services, to assess interventions, public policies, and research.
\end{abstract}

Keywords: child abuse, inventories, test validity, family violence, psychological assessment

\section{Validez de Criterio del Inventario Child Abuse Potential (CAP)}

\begin{abstract}
Resumen: Existe gran interés en el tema de la violencia contra los niños, pero son raras las formas de evaluación, lo que dificulta el desarrollo de intervenciones.El Inventario Child Abuse Potential (CAP) se considera eficaz en la identificación temprana de padres considerados en riesgo de agredir físicamente a sus hijos y buena herramienta para evaluar las intervenciones en el área de abuso contra niños. Este estudio tuvo como objetivo realizar la validez de criterio del Inventario CAP, por una comparación de sus resultados con padres de alto riesgo y padres sin riesgo para el abuso contra los niños. Participaron dos grupos: un de 20 padres que habían practicado el abuso físico y otro de 20 no agresores, del mismo nivel socioeconómico y educativo. Los resultados indicaron que el instrumento es capaz de evaluar las diferencias entre los grupos propuestos y ha demostrado ser una buena herramienta para el trabajo de los servicios de protección de la infancia, evaluación de las intervenciones y políticas públicas o de investigación.
\end{abstract}

Palabras clave: abuso de niño, inventarios, validación de test, violencia doméstica, evaluación psicológica

A violência, abuso ou maus-tratos praticados contra a criança são coibidos, no Brasil, pela Constituição Federal e pelo Estatuto da Criança e Adolescente - ECA (Lei $\mathrm{n}^{\circ} .8 .069$, de 1990) que dispõem sobre a proteção de crianças e adolescentes, até 18 anos de idade. A legislação brasileira está de acordo com as normas internacionais estabelecidas pela Convenção dos Direitos da Criança que estabelecem

\footnotetext{
1 Endereço para correspondência:

Lúcia Cavalcanti de Albuquerque Williams. Universidade Federal de São Carlos. Departamento de Psicologia. Rodovia Washington Luiz, km 235. CEP 13.565-905. São Carlos-SP.E-mail: williams@ufscar.br
}

o direito de a criança estar livre de todo tipo de violência (Svevo-Cianci, Herczog, Krappmann, \& Cook, 2011).

A Organização Mundial de Saúde e a Sociedade Internacional para Prevenção do Abuso Infantil e Negligência (World Health Organization \& International Society for Prevention of Child Abuse and Neglect, 2006) caracterizam os maus-tratos como o abuso físico, emocional e sexual e a negligência contra a criança, bem como a sua exploração, seja ela comercial ou de outro tipo, resultando em prejuízo real ou potencial à saúde, à sobrevivência, ao desenvolvimento e à dignidade da criança, em um contexto de um relacionamento de responsabilidade, confiança e 
poder. Essa será a definição adotada no presente estudo, pois carrega, em sua teoria subjacente, a ideia de que o conceito de violência envolve um padrão hierárquico de relacionamento no qual o agressor (cuidador) estaria em uma posição de superioridade em relação à vítima (criança) que, por estar em condição especial de desenvolvimento, seria mais vulnerável a qualquer tipo de agressão aos seus direitos.

O presente estudo focou-se exclusivamente na definição de abuso físico que, segundo a World Health Organization e a International Society for Prevention of Child Abuse and Neglect (2006), pode ser caracterizado pelo uso intencional da força física contra uma criança que resulte ou tem grandes chances de resultar em prejuízos à saúde, à sobrevivência, ao desenvolvimento ou à dignidade da criança. Nesta definição estão incluídos: bater, espancar, chutar, chacoalhar, morder, estrangular, escaldar, queimar, intoxicar e sufocar. Observase que a violência física é, geralmente, utilizada no contexto doméstico com o objetivo de punir ou disciplinar a criança por algum erro cometido (Carmo \& Harada, 2006; Gomes, Deslandes, Veiga, Bhering, \& Santos, 2002; Padilha \& Williams, 2004).

Supõe-se que o número de casos de maus-tratos divulgados oficialmente seja menor do que o número real de casos (Bazon, 2008; Bringiotti, 1999), estimando-se que sejam divulgados somentede $10 \%$ a $30 \%$ dos casos, tornando visível a necessidade de desenvolvimento e aperfeiçoamento das intervenções na área de violência intrafamiliar no país (Bazon, 2008; Bérgamo, 2007; Faleiros $\&$ Bazon, 2008). Sendo assim, um dos principais problemas para o desenvolvimento de intervenções refere-se ao baixo nível de identificação dos casos de violência que acabam não sendo alvo de ações de prevenção, o que aumenta ainda mais o índice de violência e, consequentemente, das estatísticas fatais (Conselho Nacional de Secretários de Saúde [CONASS], 2007).

\section{Inventário de Potencial de Abuso Infantil (CAP)}

A adaptação de instrumentos para a mensuração da violência contra crianças brasileiras torna-se importante, pois há poucos instrumentos desenvolvidos ou adaptados, no país, para avaliação de intervenções ou identificação de abuso infantil. O Child Abuse Potential Inventory (Inventário CAP) foi elaborado por Milner (1986) nos Estados Unidos, a partir de uma ampla revisão da literatura de mais de 700 livros e artigos relacionados à temática de abuso infantil na literatura (Milner \& Williams, 1978). Foi construído de modo a identificar fatores de risco associados aos maus-tratos presentes no microssistema familiar, tendo como base o modelo do Processamento da Informação Social (Milner, 1990, 1993,1994 2000, 2003) que integra fatores psicológicos e sociais vinculados à ocorrência do comportamento fisicamente abusivo dos pais.

Em sua versão atual, o Inventário CAP (Milner, 1986) é composto por 160 itens (Exemplo: "nunca sinto pena dos outros"; "gosto de ter animais domésticos"; "sempre fui saudável e forte"; "gosto da maior parte das pessoas" e "sou uma pessoa confusa"), nos quais o respondente deve assinalar com um X se "concorda" ou "discorda" da afirmação proposta. Os itens do Inventário estão agrupados em subescalas. A subescala de Abuso é considerada a principal escala e é composta por 77 itens. Por sua vez, a escala de Abuso está subdividida em seis subescalas, a saber: (1) Sofrimento; (2) Rigidez; (3) Infelicidade; (4) Problemas com a criança e consigo mesmo; (5) Problemas com a família e (6) Problemas com os outros. Adicionalmente, o CAP contém três escalas de validade: Mentira (18 itens), Respostas ao Acaso (18 itens) e Inconsistência (20 pares de itens). Os escores das três escalas de validade geram três índices de distorção da resposta: Respostas ao Acaso, Imagem Positiva de si mesmo e Imagem Negativa de si mesmo.

O Inventário CAP é útil como instrumento de triagem, avaliando o potencial ou risco de os pais maltratarem seus filhos, por meio da identificação de algumas características psicossociais correlacionadas ao fenômeno da violência contra a criança, dando maior ênfase ao abuso físico. Relacionado com outros métodos de mensuração (como entrevistas, observações diretas e testes, escalas e inventários), o Inventário CAP pode servir tanto para mapear diferenças entre grupos de indivíduos quanto para classificar individualmente os casos (Milner, 1986). A importância do instrumento pode ser destacada pela adaptação do mesmo em diversos continentes, como Europa, Ásia e América do Sul.

O estudo desenvolvido na Bélgica (Grietens, De Haene, \& Uyttebroek, 2007) procurou verificar a fidedignidade e a validade convergente do Inventário CAP, obtendo como resultado índices altos de consistência interna e correlação entre o Inventário CAP e outros instrumentos relacionados ao cuidado infantil e estresse parental. Na Croácia (Pecnik \& Ajdukovic, 1995), os pesquisadores realizaram a análise discriminante e o cálculo de consistência interna do CAP. A análise discriminante indicou uma classificação correta da subescala de Abuso de 87,59\% e um coeficiente de consistência interna de 0,91. Na Espanha (De Paul, Arruabarrena, \& Milner, 1991), os pesquisadores apresentaram um índice de $91,7 \%$ de classificação correta dos casos para a análise discriminante, e a análise fatorial apresentou uma estrutura fatorial similar a do Inventário CAP original, composta por seis fatores. No estudo desenvolvido na Grécia (Diareme, Tsiantis, \& Tsitoura, 1997), o Inventário CAP demonstrou um índice de 0,91 de fidedignidade, uma estrutura fatorial semelhante à versão original do instrumento e um índice de $78,1 \%$ de classificação correta para análise discriminante. Na China (Chan, Lam, Chun, \& So, 2006), os resultados da pesquisa apresentaram um índice de fidedignidade de 0,90 pelo coeficiente alfa, a análise fatorial apresentou uma estrutura fatorial de seis fatores (semelhante à versão original do instrumento) e verificaram-se bons indicadores de validade concorrente do inventário.

Os resultados da pesquisa sobre o CAP, realizada na Argentina (Bringiotti, Barbich, \& Del Paul, 1998), indicaram 
um alto índice de fidedignidade do instrumento $(0,94)$ e indicações positivas da capacidade preditiva e discriminante do inventário. No Chile (Haz \& Ramirez, 2002), o Inventário CAP apresentou alta consistência interna $(0,95)$, por meio do Alfa de Cronbach, e a análise discriminante apresentou um índice moderado de $70 \%$ de classificação correta dos casos.

No Brasil, Rios (2010) realizou a adaptação transcultural do Inventário CAP com os objetivos específicos de realizar e avaliar a equivalência semântica do instrumento, sua validade de conteúdo e sua validade de construto. Em relação à validade de construto, participaram do estudo 135 cuidadores (selecionados por amostragem aleatória simples) que responderam ao Inventário $\mathrm{CAP}$ e a um questionário sociodemográfico. Em relação ao escore de potencial para abuso físico infantil, a média de escore da amostra brasileira foi de 180,1 $(D P=102,5)$, resultado superior ao encontrado na amostra norte-americana $(M=91,0$ e $D P=75,0)$. Por meio do Alfa de Cronbach, chegou-se ao coeficiente de 0,95 de consistência interna.

Bérgamo, Pasian, Mello e Bazon (2009) verificaram a consistência interna do Inventário CAP, em sua versão brasileira (Ávila de Mello et al., 2008), e sua capacidade de discriminar um grupo clínico de cuidadores $(n=60)$ notificados em Conselhos Tutelares de um grupo de comparação de cuidadores $(n=60)$ sem histórico de maustratos com os filhos. Segundo análise pelo coeficiente Alpha de Cronbach, o índice de fidedignidade para a amostra total $(n=120)$ foi superior a 0,90 . A análise discriminante foi realizada a partir da comparação da distribuição dos resultados para cada item do Inventário CAP, utilizando-se o teste qui-quadrado ou o Teste Exato de Fisher $(p \leq 0,05)$. Os resultados da análise discriminante demonstraram que, dos 77 itens da subescala de Abuso, 35 itens discriminaram o grupo clínico do grupo de comparação.

O estudo de mestrado de Bérgamo (2007) teve por objetivo verificar a relação entre fatores de risco associados à figura do cuidador com os maus-tratos físicos. O Inventário CAP (Ávila de Mello et al., 2008) foi aplicado em dois grupos ( $n=30$ em cada grupo), sendo um grupo clínico e outro de comparação. Adicionalmente à aplicação do Inventário CAP, outros instrumentos foram aplicados na amostra (Índice de Estresse Parental [ISP], Inventário de Estilos Parentais [IEP], Questionário de Apoio Social [QAS], Entrevista da História da Infância do Adulto e Questionário de Caracterização Sociodemográfica). Para análise de dados, utilizaram-se estatísticas descritivas, teste $t$ Student, teste Mann Whitney Rank-sum, teste Qui-quadrado e Exato de Fisher. Como resultados, observou-se que o grupo clínico apresentou diferenças significativas $(p \leq 0,05)$ em relação ao grupo de comparação para as variáveis angústia, rigidez, problemas com a criança e consigo mesmo e problemas com os outros.

Ávila de Mello (2008) verificou os fatores de risco relativos à figura do cuidador que estariam relacionados com a presença de negligência. Foram comparados dois grupos de pais/cuidadores, recorrendo ao Inventário CAP (Ávila de
Mello et al., 2008) como estratégia avaliativa, sendo que o grupo clínico foi formado por 30 pais/cuidadores notificados ao Conselho Tutelar como sendo negligentes e o grupo de comparação, composto por 30 pais/cuidadores sem histórico de negligência com seus filhos, retirados por indicação da comunidade, constituindo uma amostra por conveniência. Os dados do Inventário CAP identificaram diferenças de escore significativas $(p \leq 0,05)$ entre grupo clínico e de comparação, indicando que o grupo clínico possuía alto potencial de risco para abuso infantil.

Finalmente, Piñon (2008) buscou caracterizar uma amostra representativa $(n=433)$ da cidade de Ribeirão Preto-SP em termos de potencial de risco para os maustratos e a possível associação com as variáveis apontadas na literatura como fatores de risco para os mesmos. Além do Inventário CAP (Ávila de Mello et al., 2008), outros instrumentos foram aplicados na amostra (ISP, IEP, QAS, Entrevista da História da Infância do Adulto e Questionário de Caracterização Sociodemográfica). Para análise de dados, utilizaram-se estatísticas descritivas, teste $t$ Student e correlação $r$ de Pearson. As variáveis sociodemográficas, de estilos parentais e estresse parental foram relacionadas com os resultados do Inventário CAP.

Os principais resultados indicaram que: (a) cuidadores com alto potencial de abuso físico apresentaram razoável correlação negativa com nível de escolaridade $(r=-0,36$; $p<0,001$ ); (b) cuidadores com alto potencial de abuso físico apresentaram razoável correlação positiva com número de filhos ( $r=0,32 ; p<0,001)$; (c) cuidadores masculinos apresentaram média de potencial para abuso infantil superior à média das cuidadoras do sexo feminino $(p=0,008)$; (d) cuidadores(as) solteiros(as) apresentaram médias de potencial para abuso infantil superiores à média de cuidadores(as) casados(as) ( $p<0,001)$; (e) cuidadores(as) sem emprego apresentaram média de potencial para abuso infantil superior à média de cuidadores(as) empregados ( $p<0,001)$; (f) cuidadores com alto potencial de abuso físico apresentaram razoável correlação positiva com o estilo parental caracterizado por punição inconsistente $(r=0,31 ; p<0,001)$; (g) cuidadores com alto potencial de abuso físico apresentaram razoável correlação positiva com o estilo parental caracterizado por negligência $(r=0,33$; $p<0,001$ ); (h) cuidadores com alto potencial de abuso físico apresentaram razoável correlação positiva com o estilo parental caracterizado por disciplina relaxada $(r=0,34$; $p<0,001)$ e (i) cuidadores com alto potencial de abuso físico apresentaram correlação positiva com estresse parental percebido $(r=0,50 ; p<0,001)$.

Nota-se que pesquisas utilizando o Inventário CAP (em outros países e no Brasil) apresentaram resultados sinalizadores da validade deste instrumento, possibilitando identificar grupos de pais que maltratam e não maltratam seus filhos e sua convergência de dados quando relacionados a outros instrumentos relacionados à violência intrafamiliar. Nesse sentido, o presente estudo objetivou realizar a validade 
de critério do Inventário CAP, por meio da comparação entre dois grupos de pais, sendo um grupo composto por 20 pais denunciados por agressões físicas e o outro grupo de 20 pais não agressores de igual nível socioeconômico e educacional.

\section{Método}

\section{Participantes}

Participaram do estudo dois grupos de cuidadores, sendo um grupo considerado clínico e um grupo de comparação. O grupo clínico (denominado CT) foi composto por 20 cuidadores que haviam sido notificados no Conselho Tutelar da cidade de São Carlos-SP por cometerem abuso físico, durante os seis meses anteriores à coleta de dados da pesquisa, sendo dois cuidadores do sexo masculino e 18 cuidadoras do sexo feminino, com idade média de 34 anos $(D P=9,8)$, com a média de 7,1 anos de educação $(D P=3,1)$. No $C T, 12$ cuidadores eram casados, sendo o número médio de filhos por família igual a 2,8 , enquanto a idade média da criança foi de 10,35 $(D P=2,3)$. $O$ grupo de comparação (denominado ESC) foi composto por 20 cuidadores selecionados em duas escolas públicas municipais, sendo um cuidador do sexo masculino e 19 cuidadoras do sexo feminino, com idade média de 38,6 anos $(D P=6,0)$, com a média de 8,6 anos de educação $(D P=2,7)$. No ESC, 18 cuidadores eram casados, existindo número médio de filhos por família igual a 1,9 $(D P=2,7)$, com idade média da criança de 10,6 $(D P=2,2)$ anos. Houve diferenças significativas apenas para as variáveis idade $(t(39)=1,22 ; p<0,24)$ e estado civil $\left(\chi^{2}=5,7 ; p<0,32\right)$, entre os dois grupos.

\section{Instrumentos}

1) Inventário CAP, elaborado por Milner (1986). Foi utilizada a versão do Inventário CAP em português (Brasil) elaborada e validada por Ávila de Mello et al. (2008). Embora o instrumento seja padronizado na forma de questionário, para ser lido e respondido por cada participante, no presente estudo ele foi aplicado sob a forma de entrevista, ou seja, o pesquisador lia em voz alta o item do CAP, e o participante indicava a alternativa escolhida. Escolheu-se a aplicação do instrumento pelo procedimento de entrevista, pois foi observado que a baixa escolaridade dos participantes dificultava a compreensão dos itens.

2) Checklist de Identificação de Crianças não Abusadas: adaptado da Cartilha Epidemiológica (Bringiotti, 2000), traduzida para o português (Brasil) e adaptada por Faleiros e Bazon (2008) e Matias e Bazon (2005), para que os professores identificassem as crianças não abusadas. $\mathrm{O}$ Checklist apresenta uma definição de cada tipo de maustratos (maus-tratos físicos, abandono físico, abuso sexual, maus-tratos emocionais, abandono emocional, mendicidade, trabalho infantil, corrupção, participação da criança em ações delituosas com sua família e falta de controle parental sobre a conduta dos filhos). $\mathrm{O}$ instrumento contém 26 sentenças de características das crianças (por exemplo: confia em outros e cria relações afetivas com outros - parece não ter dificuldade em se apegar) e 18 sentenças de características da família (por exemplo: desemprego/instabilidade no trabalho dos adultos responsáveis).

3) Critério de Classificação Econômica Brasil (CCEB): O CCEB foi elaborado pela Associação Nacional de Empresas de Pesquisa (ANEP), a partir de um estudo realizado com base no banco de dados do Levantamento Socioeconômico de 2000 do Instituto Brasileiro de Opinião Pública e Estatística (IBOPE). O CCEB tem por objetivo caracterizar os indivíduos segundo seu poder aquisitivo, ou seja, classificá-los quanto às classes socioeconômicas, de acordo com a posse de objetos ou bens de consumo e o grau de instrução do chefe da família. As classes vão de A1 a E, e os indivíduos são classificados em cada uma delas, conforme o total de pontos alcançados, os quais variam de 0 a 34 .

\section{Procedimento}

Coleta de dados. Para o início da coleta de dados, a equipe de pesquisa entrou em contato com o Conselho Tutelar da cidade, a fim de triar os participantes do grupo de pais considerados de alto risco para o abuso infantil. As conselheiras deliberaram como seria o procedimento de escolha dos participantes, visto que a pesquisadora não poderia entrar em contato com os prontuários e tampouco com os casos. Assim, as conselheiras forneceram apenas nomes e telefones de cuidadores notificados por haverem cometido abuso físico contra alguma criança sob seus cuidados. De posse desses dados, a equipe de pesquisa entrou em contato, por telefone, com cada participante explicitando-se os objetivos da pesquisa e examinando a disponibilidade e interesse dos mesmos em participar da mesma, até que o número de participantes fosse completado. Caso o participante aceitasse fazer parte da pesquisa, era solicitado o fornecimento do endereço de sua residência, e uma data era marcada para visita da equipe de pesquisa. A coleta de dados foi realizada na casa dos cuidadores/ participantes do estudo. Na ocasião da coleta de dados, era inicialmente lido o Termo de Consentimento Livre e Esclarecido (TCLE) e explicado verbalmente o conteúdo do mesmo. Em seguida, o cuidador era questionado se havia alguma dúvida ou comentário, formalizando-se o aceite para participação da pesquisa com a assinatura do participante. Finalmente, era aplicado o Inventário CAP, na forma de entrevista, com o cuidador que havia sido notificado ao Conselho Tutelar.

Concomitantemente, e mediante autorização da Secretaria de Educação do Município, a equipe de pesquisa entrou em contato com duas escolas selecionadas para a coleta de dados, visto que a maior parte das crianças do CT estudava nessas escolas. Nelas, buscou-se contato com os professores que atendiam alunos na mesma faixa etária das crianças do CT, e a equipe de pesquisa solicitava que os professores identificassem, em suas salas de aula, 
por meio do Checklist de Identificação de Crianças não Abusadas, as crianças que não indicassem sofrer agressões físicas pelos cuidadores. Optou-se por selecionar o ESC por meio dos professores porque é no contexto escolar que é identificada a maioria dos casos de maus-tratos (Vagostello, Oliveira, Silva, Donofrio, \& Moreno, 2003). Depois de identificadas tais crianças, as escolas forneceram os dados dos cuidadores, como, por exemplo, idade, sexo, escolaridade e endereço para que a equipe de pesquisadores os localizasse e verificasse o interesse em participar do estudo. A equipe de pesquisadores entrou em contato com os cuidadores indicados até que se completasse um grupo de 20 participantes. O procedimento de coleta de dados com tais cuidadores foi igual ao do CT, ou seja, contato telefônico e posterior visita, assinatura de TCLE, seguida da aplicação do Inventário CAP. Em ambos os grupos, o tempo médio de duração da sessão de coleta de dados com cada participante foi de 60 minutos.

Análise de dados. Para análise dos resultados do Inventário CAP, utilizou-se o software CAPSCORE, cedido pelo autor para uso exclusivo dos pesquisadores. O CAPSCORE calcula a somatória dos pesos de cada um dos itens do inventário, fornecendo um escore geral para o potencial de abuso infantil, escores para cada uma das subescalas e para as escalas de validade. $\mathrm{O}$ escore geral de potencial de abuso pode variar entre 0 e 486 , sendo 215 a nota de corte na normatização norte-americana. Após cálculo do escore para todas as escalas, procedeu-se ao cálculo da média e do desvio-padrão dos escores para a amostra estudada. Para verificar as diferenças de escore do Inventário CAP entre os dois grupos, utilizou-se o teste U de Mann-Whitney. Considerando a ausência de normas brasileiras, no presente estudo, assim como em outros estudos brasileiros (Bérgamo, 2007; Bérgamo et al., 2009; Piñon, 2008; Rios, 2010; Rios, Williams, Schelini, Bazon \& Piñon, 2013), utilizaram-se as notas de corte normatizadas nos Estados Unidos da América, a título de comparação.

\section{Considerações Éticas}

O presente estudo foi aprovado pelo Comitê de Ética em Pesquisa com Seres Humanos da Universidade Federal de São Carlos (CEP/UFSCar) sob o número de protocolo CAAE 3268.0.000.135-07.

\section{Resultados e Discussão}

Em relação ao escore do Inventário CAP para os dois grupos, observou-se uma média de escore mais elevada no CT $(M=265,5 ; D P=93,1)$ em relação ao $\operatorname{ESC}(M=103,4$; $D P=53,1)$. Essa diferença de escore entre grupos foi estatisticamente significativa, segundo o teste $\mathrm{U}$ de MannWhitney $(p=0,0001 ; z=-4,49)$.

Tabela 1

Escores Médios e Desvios-Padrão para as Escalas do Inventário CAP para os Grupos Clínico (CT) e de Comparação (ESC)

\begin{tabular}{|c|c|c|c|c|c|}
\hline \multirow[b]{2}{*}{ Escalas } & \multirow[b]{2}{*}{ Nota de corte ${ }^{\mathrm{a}}$} & \multicolumn{2}{|c|}{$\begin{array}{c}\text { ESC } \\
n=20\end{array}$} & \multicolumn{2}{|c|}{$\begin{array}{c}\text { CT } \\
n=20\end{array}$} \\
\hline & & $M$ & $D P$ & $M$ & $D P$ \\
\hline Abuso & 215 & $103,4^{*}$ & 53,1 & $265,5^{*}$ & 93,1 \\
\hline Sofrimento & 152 & $50 * *$ & 37,3 & $50 * *$ & 37,3 \\
\hline Rigidez & 30 & $29,9 * *$ & 17,6 & $29,9 * *$ & 17,6 \\
\hline Infelicidade & 23 & $8,95 * *$ & 7,2 & $8,95 * *$ & 7,2 \\
\hline Problemas com criança & 11 & $3,05^{* *}$ & 4,17 & $1,6^{* *}$ & 3,52 \\
\hline Problemas com família & 18 & $1,6^{* *}$ & 3,52 & $3,05^{* *}$ & 4,17 \\
\hline Problemas com outros & 20 & $9,95 * *$ & 6 & $9,95 * *$ & 6,0 \\
\hline Mentira & 7 ou 8 & $11,2 * *$ & 2,73 & $9,57 * *$ & 3,31 \\
\hline Resposta Randômica & 6 & $3 * *$ & 1,41 & $2,67 * *$ & 1,59 \\
\hline Inconsistência & 6 & $4,45 * *$ & 2,06 & $6,43 * *$ & 2,18 \\
\hline
\end{tabular}

Nota. ${ }^{a}$ Segundo nota de corte norte-americana. $* p=0,0001 ; * * p<0,05$

De acordo com o Manual do Inventário CAP (Milner, 1986), a nota de corte norte-americana para o instrumento corresponde ao valor de 215 pontos. A partir deste parâmetro normativo, o CT apresentou valores médios superiores $(265,5)$, sugerindo que este grupo, do atual estudo, apresentava alto potencial de risco para abusar fisicamente seus filhos. Por outro lado, o ESC apresentou valores abaixo da nota de corte proposta no manual do Inventário CAP, indicando baixo potencial de risco dos cuidadores para abusar suas crianças. Em relação ao Brasil, comparando-se os resultados do presente estudo com os dados da pesquisa realizada por Bérgamo (2007), que verificou a capacidade de discriminação do Inventário CAP quando aplicado a dois grupos distintos (clínico e de comparação, ambos com 
$n=30$ ), notou-se que tal autora também encontrou diferenças significativas entre os grupos, sendo que a média do escore geral do grupo clínico foi de $166,90(D P=83,34)$ e do grupo de comparação 223,87 $(D P=99,30)$.

Além disso, Bérgamo (2007) analisou cada uma das subescalas, encontrando diferenças significativas $(p \leq 0,05)$ para as seguintes subescalas: angústia, rigidez, problemas com a criança e consigo mesmo, problemas com os outros, o que corrobora a hipótese inicial de seu estudo sobre a capacidade do instrumento em discriminar os grupos contrastantes em termos de maus-tratos. Em estudos internacionais que trabalharam com a validade de critério do Inventário CAP em países como Croácia (Pecnik \& Ajdukovic, 1995), Espanha (De Paul et al., 1991), Argentina (Bringiotti et al., 1998) e Chile (Haz \& Ramirez, 2002), a diferença entre as médias de escores entre os grupos de cuidadores considerados de alto risco para o abuso infantil e do grupo de cuidadores considerados de baixo risco também demonstrou ser estatisticamente significativa, indicando que o Inventário CAP é sensível para discriminar grupos de cuidadores de alto e baixo risco para o abuso infantil.

Adicionalmente, em estudos brasileiros com amostras gerais da população, selecionadas de forma aleatória, encontrou-se em um estudo a média de escore geral de 180,1 $(D P=102,5)$ para uma amostra de 135 cuidadores (Rios et al., 2013). Em outro estudo, a média de escore geral foi 182 $(D P=96,46)$ para uma amostra de 433 cuidadores (Piñon, 2008).

Pode-se notar que, em todas as subescalas, a diferença de escore entre grupos foi estatisticamente significativa, segundo o teste $\mathrm{U}$ de Mann-Whitney $(p=0,05)$. Comparandose os escores médios do CT em relação ao ESC, os primeiros sinalizaram indicadores de maior sofrimento psíquico $(M=161,0$ e $D P=68,4 ; M=50,0$ e $D P=37,3)$, maior rigidez $(M=43,9$ e $D P=19,5 ; M=29,9$ e $D P=17,6)$, maior infelicidade $(M=17,8$ e $D P=11,9 ; M=8,95$ e $D P=7,2)$ e tinham mais problemas com a família $(M=9,35$ e $D P=6,61$; $M=1,6$ e $D P=3,52)$, com os outros $(M=19,6$ e $D P=5,7$; $M=9,95$ e $D P=6,0)$ e problemas com as crianças e consigo mesmo $(M=13,9$ e $D P=13,5 ; M=3,05$ e $D P=4,17)$.

Tendo como base esses referenciais normativos norteamericanos, nota-se que a média de escore para CT, obtida no presente estudo, para as subescalas de sofrimento, rigidez e problemas com a criança e consigo mesmo apresentou índices acima das notas de corte norte-americanas. Por outro lado, o ESC não apresentou médias de escore superiores ao ponto de corte utilizado nos Estados Unidos da América. Tais dados apontam para a validade do Inventário CAP, na medida em que se mostra empiricamente capaz de discriminar grupos de pais considerados abusadores de grupo de pais considerados não agressores.

Na mesma direção dos resultados do presente estudo, Bérgamo (2007) identificou que o grupo clínico apresentou médias superiores de escore para as subescalas de sofrimento e rigidez quando comparado ao grupo de comparação. O grupo de comparação não apresentou média de escore acima da nota de corte proposta por Milner (1986), enquanto no grupo clínico apenas a subescala Rigidez $(M=42,53 ; D P=16,37)$ apresentou valores acima da nota de corte norte-americana.

Em outro estudo realizado em São Carlos-SP com uma amostra representativa da população, Rios et al., 2013. encontraram índices acima da nota de corte norte-americana para a subescala Rigidez com média de escore de 41,8 $(D P=15,2)$, em um universo de 135 cuidadores. Piñon (2008) também encontrou índices acima da nota de corte norteamericana para a subescala Rigidez com média de escore de $34(D P=16,67)$ para uma amostra representativa de 433 cuidadores de um bairro da cidade de Ribeirão Preto-SP.

Os dados da subescala Rigidez, tanto no presente estudo como no estudo de Bérgamo (2007), Piñon (2008) e Rios et al., 2013, chamam a atenção por apresentarem média de escore relativamente mais alta, em ambos os grupos de cuidadores, quando comparada às outras subescalas. Esses dados podem ser analisados sob a ótica do modelo do Processamento da Informação Social (Milner, 2003), base do Inventário CAP, que integra fatores psicológicos e sociais vinculados com a ocorrência do comportamento abusivo dos pais. De acordo com a teoria cognitiva, detalhes do contexto social são internalizados na forma de esquemas pelo indivíduo. Essas estruturas de informação internalizadas influenciariam percepções, interpretações e reações subsequentes na vida social do indivíduo (Farc, Crouch, Skowronski, \& Milner, 2008). Assim, poder-se-ia supor que os cuidadores brasileiros apresentam percepções mais rígidas sobre seus filhos, em virtude da visão cultural de posse da criança ou pela violência na mídia (Cecconnello, De Antoni, \& Koller, 2003). Futuros estudos poderiam aprofundar tais colocações.

Em relação às escalas de validade do Inventário CAP, o escore médio referente à escala de Mentira encontrado no ESC foi maior $(M=11,2 ; D P=2,73)$ do que o encontrado no $\mathrm{CT}$ $(M=9,57 ; D P=3,31)$. O mesmo ocorreu com o escore médio da escala Resposta Randômica, comparando-se resultados do $\operatorname{ESC}(M=3 ; D P=1,41)$ e do CT $(M=2,67 ; D P=1,59)$. Porém, em relação à escala Inconsistência, o grupo ESC teve escore médio inferior $(M=4,45 ; D P=2,06)$ quando comparado ao índice encontrado no CT $(M=6,43 ; D P=2,18)$. Segundo as notas de corte propostas no manual do Inventário CAP (Milner, 1986), tanto o CT quanto o ESC apresentaram médias de escore acima da nota de corte norte-americana para a escala Mentira, e apenas o CT apresentou média de escore acima da nota de corte para escala Inconsistência. Semelhantemente, no trabalho de Bérgamo (2007), Piñon (2008) e Rios (2010), os escores médios encontrados para a escala Mentira, tanto para o CT quanto para o ESC, foram acima da nota de corte norteamericana proposta por Milner (1986).

Hipotetiza-se que os escores altos para a escala Mentira devem-se ao fato de que o Inventário CAP teve sua aplicação na forma de entrevista, ou seja, o pesquisador lia cada item, e o respondente dizia verbalmente se concordava ou não com a afirmação, e em seguida o próprio 
pesquisador marcava as respostas do participante na folha de registro dos dados. Por se tratar de um instrumento que avalia percepções e crenças dos respondentes, supõe-se que a aplicação na forma de entrevista facilitaria a exposição de defesas do participante que poderia dar respostas socialmente aceitáveis, ao invés de revelar suas verdadeiras percepções, a fim de evitar o julgamento do pesquisador. Tal hipótese poderia ser testada em um estudo que comparasse tal escala de validade em grupos que apresentassem formas diferentes de aplicação do Inventário.

Com relação ao modo de aplicação na forma de entrevista, Milner (1986) afirma que, apesar de não existirem dados empíricos a respeito dos efeitos da leitura dos itens do Inventário CAP, sendo necessário pode-se efetivá-lo na forma de entrevista, quando os itens devem ser lidos em voz alta, sem explicações, conselhos, ou comentários e ocorrendo em um lugar privado. No presente estudo, procurou-se seguir as orientações de aplicação colocadas pelo autor do instrumento, mas a despeito de tal procedimento, os índices da escala Mentira mantiveram-se elevados. Sugere-se que, em função das peculiaridades de aplicação do Inventário no Brasil, sejam elaborados estudos que procurem normatizar o Inventário CAP, elaborando notas de corte de cada subescala, baseadas na cultura brasileira.

A partir dos escores das escalas de validade, é possível calcular três índices de distorção da resposta: Imagem positiva de si mesmo (quando a escala Mentira tem escore maior ou igual a 7 e a escala Randômica menor ou igual a 5), Imagem negativa de si mesmo (quando a escala Resposta Randômica é maior ou igual a 6 , e a escala Inconsistência é menor ou igual a 5) e Respostas ao Acaso (quando a escala Randômica é maior ou igual a 6 , e a escala Inconsistência é maior ou igual a 6). No presente estudo, o índice de distorção Imagem positiva de si mesmo foi o maior em ambos os grupos $-\mathrm{CT}=13 \mathrm{e}$ $\mathrm{ESC}=16-$, sugerindo que os participantes forneceram respostas consideradas socialmente aceitáveis.

Fato interessante foi que, tanto na presente pesquisa, como na pesquisa de Bérgamo (2007), o ESC apresentou maiores escores para a escala Mentira e para o índice de distorção Imagem positiva de si mesmo, quando comparado aos escores do CT. Isso poderia indicar que, no grupo considerado sem potencial para abuso infantil, houve maior número de respostas socialmente aceitáveis.

\section{Considerações Finais}

A partir dos dados da presente pesquisa, pode-se afirmar que as diferenças de escore entre o CT e o ESC foram estatisticamente significativas e reforçam os dados apresentados por Milner (1986) e corroborados, no Brasil, por Bérgamo (2007), constituindo-se em evidências empíricas de validade de critério para o Inventário CAP. Isso significa que o instrumento possui indicadores de refinamento e constituição técnica suficiente para avaliar possíveis diferenças entre os grupos presentemente avaliados.

Como limitações do estudo, pontua-se que o tamanho da amostra foi reduzido e que a amostra dos dois grupos foi constituída por casos extremos, ou seja, a metodologia usada para triagem da amostra favoreceu a seleção de participantes considerados gravíssimos para o CT e participantes considerados de extremo baixo risco para abuso infantil para o ESC. Sugerem-se outros estudos com maior número de participantes e com amostras consideradas mais homogêneas para a variável do abuso infantil, a fim de verificar o refinamento do instrumento para distinguir grupos compostos por casos considerados mais moderados, sendo estes os casos com maior dificuldade de identificação. Por fim, sugere-se o desenvolvimento de outros estudos com o Inventário CAP para verificação de sua validade e posterior normatização para o Brasil.

\section{Referências}

Ávila de Mello, I. L. M. (2008). Negligência de crianças: Contribuições para a avaliação de fatores de risco psicossociais. Dissertação de mestrado não publicada, Universidade São Paulo, Ribeirão Preto, SP.

Ávila de Mello, I. L. M., Bérgamo, L. P. D., Piñon, E. A., Rios, K. S. A., Williams, L. C. A., \& Bazon, M. R. (2008). Inventário de Potencial de Abuso Infantil- CAP: Versão em Português. Instrumento não publicado.

Bazon, M. R. (2008). Violências contra crianças e adolescentes: Análise de quatro anos de notificações feitas ao Conselho Tutelar na cidade de Ribeirão Preto, São Paulo, Brasil. Cadernos de Saúde Pública, 24(2), 323-332. doi:10.1590/S0102-311X2008000200011

Bérgamo, L. P. D. (2007). Maus-tratos físicos de crianças: Contribuições para a avaliação de fatores de risco psicossociais. Dissertação de mestrado não publicada, Universidade São Paulo, Ribeirão Preto, SP.

Bérgamo, L. P. D., Pasian, S. R., Mello, I. L. M. A., \& Bazon, M. R. (2009). O Inventário de Potencial de Maustratos infantil: Estudo de precisão e validade. Avaliação Psicológica, 8(3), 425-435.

Bringiotti, M. I. (1999). Maltrato infantil: Factores de riesgo para el maltrato físico en la población infantil que concurre a las escuelas dependientes del Gobierno de la Ciudad de Buenos Aires. Madrid, España: Niño e Dávila.

Bringiotti, M. I. (2000). La escuela ante los niños maltratados. Buenos Aires, Argentina: Paidós.

Bringiotti, M. I., Barbich, M. I. B., \& De Paul, J. (1998). Validación de una versión preliminar del Child Abuse Potential Inventory para su uso en Argentina. Child Abuse \& Neglect, 22(9), 881-888.

Carmo, C. J., \& Harada, M. J. C. S. (2006). Violência física como prática educativa. Revista LatinoAmericana de Enfermagem, 14(6), 849-856. doi:10.1590/S0104-11692006000600004

Cecconello, A. M., De Antoni. C. \& Koller, S. H. (2003). Práticas educativas parentais e o abuso físico no contexto familiar. Psicologia em Estudo, 8 (n. esp.), 45-54. doi: $10.1590 /$ S1413-73722003000300007 
Chan, Y. C., Lam, G. L. T., Chun, P. K. R., \& So, M. T. E. (2006). Confirmatory factor analysis of the Child Abuse Potential Inventory: Results based on a sample of Chinese mothers in Hong Kong. Child Abuse \& Neglect, 30(9), 1005-1016. doi:10.1016/j.chiabu.2006.05.005

Conselho Nacional de Secretários de Saúde. (2007). Violência: Uma epidemia silenciosa. Brasília: CONASS.

De Paul, J., Arruabarrena, I., \& Milner, J. S. (1991).Validación de una versión española del Child Abuse Potential Inventory para su uso en España. Child Abuse \& Neglect, 15(4), 495-504. doi:10.1016/0145-2134(91)90033-A

Diareme, S., Tsiantis, J., \& Tsitoura, S. (1997). Cross-cultural validation of the Child Abuse Potential Inventory in Greece: A preliminary study. Child Abuse \& Neglect, 21(11), 1067-1079. doi:10.1016/S0145-2134(97)00066-5

Faleiros, J. M., \& Bazon, M. R. (2008). Prevalência de maustratos em crianças de $1^{\mathrm{a}}$ a $4^{\mathrm{a}}$ série da cidade de Ribeirão Preto-SP. Paidéia (Ribeirão Preto), 18(40), 341-354. doi:10.1590/S0103-863X2008000200011

Farc, M. M., Crouch, J. L., Skowronski, J. J., \& Milner, J. S. (2008). Hostility ratings by parents at risk for child abuse: Impact of chronic and temporary schema activation. Child Abuse \& Neglect, 32(2), 177-193. doi:10.1016/j.chiabu.2007.06.001

Gomes, R., Deslandes, S. F., Veiga, M. M., Bhering, C., \& Santos, J. F. C. (2002). Por que as crianças são maltratadas?: Explicações para a prática de maus-tratos infantis na literatura. Cadernos de Saúde Pública, 18(3), 707-714.

Grietens, H., De Haene, L., \& Uyttebroek, K. (2007). Cross-cultural validation of the Child Abuse Potential Inventory in Belgium (Flanders): Relations with demographic characteristics and parenting problems. Journal of Family Violence, 22(4), 223-229. doi:10.1007/s10896-007-9074-2

Haz, A. M., \& Ramírez, V. (2002). Adaptación del Child Abuse Potential Inventory en Chile: Análisis de las dificultades y desafíos de su aplicación a partir de dos estudios Chilenos. Child Abuse \& Neglect, 26(5), 481495. doi:10.1016/S0145-2134(02)00324-1

Lei No. 8.069, de 13 de julho de 1990. (1990, 16 de julho). Dispõe sobre o Estatuto da Criança e do Adolescente e dá outras providências. Diário Oficial da União, seção 1.

Matias, A. S. A., \& Bazon, M. R. (2005). Maus-tratos de crianças de 0 a 6 anos que frequentam estabelecimentos de educação infantil na cidade de Ribeirão Preto. In Programa de Pós-graduação em Psicologia: Livro de artigos (Vol. 2, pp. 287-298). Ribeirão Preto, SP: Legis Summa.

Milner, J. S. (1986). The Child Abuse Potential Inventory: Manual (2nd ed.). Webster, NC: Psytec.

Milner, J. S. (1990). An interpretive manual for the Child Abuse Potential Inventory. Webster, NC: Psytec.

Milner, J. S. (1993). Social information processing and physical child abuse. Clinical Psychology Review, 13(3), 275-294. doi:10.1016/0272-7358(93)90024-G
Milner, J. S. (1994). Assessing physical child abuse risk: The Child Abuse Potential Inventory. Clinical Psychology Review, 14(6), 547-583. doi:10.1016/0272-7358(94)90017-5

Milner, J. S. (2000). Social information processing and child physical abuse: Theory and research. In R. A. Dienstbier (Series Ed.) \& D. J. Hansen (Vol. Ed.), Nebraska Symposium on Motivation: Vol. 46. Motivation and child maltreatment (pp. 39-84). Lincoln, NE: University of Nebraska Press.

Milner,J.S.(2003). Social information processinginhigh-risk and physically abusive parents. Child Abuse \& Neglect, 27(1), 7-20. doi:10.1016/S0145-2134(02)00506-9

Milner, J. S., \& Williams, P. P. (1978). Child abuse and neglect: A bibliography. Journal Supplement Abstract Services: Catalogof Selected Documents in Psychology, 8,42 .

Padilha, M. G. S., \& Williams, L. C. A. (2004). Considerações sobre estilos parentais de pais que cometem abuso físico, psicológico ou sexual contra seus filhos. In M. Z. S. Brandão, F. C. S. Conte, F. S. Brandão, Y. K. Ingberman, V. L. M. Silva, \& S. M. Oliani (Orgs.), Sobre comportamento e cognição: Estendendo a psicologia comportamental e cognitiva aos contextos da saúde, das organizações, das relações pais e filhos e das escolas (Vol. 14, pp. 286-291). Santo André, SP: ESETec.

Pecnik, N., \& Ajdukovic, M. (1995). The Child Abuse Potential Inventory: Cross-validation in Croatia. Psychological Reports, 76(3 Pt 1), 979-985.

Piñon, E. A. (2008). Os maus-tratos de crianças $e$ adolescentes na comunidade: $O$ potencial de risco $e$ os fatores sócio-comunitários associados. Dissertação de mestrado não publicada, Universidade São Paulo, Ribeirão Preto, SP.

Rios, K. S. A. (2010). Inventário de Potencial de Abuso Infantil - CAP: Adaptação transcultural para o Brasil. Tese de doutorado não publicada, Universidade Federal de São Carlos, São Carlos, SP.

Rios, K. S. A., Williams, L. C. A., Schelini, P. W., Bazon, M. R., \& Piñon, E. A. (2013). Inventário de Potencial de Abuso Infantil - CAP: Evidências de Validade e precisão. Avaliação Psicológica, 12(1), 51-60.

Svevo-Cianci, K. A., Herczog, M., Krappmann, L., \& Cook, P. (2011). The new UN CRC General Comment 13: "The right of the child to freedom of all forms of violence" - Changing how the world conceptualizes child protection. Child Abuse \& Neglect, 35(12), 979989. doi:10.1016/j.chiabu.2011.09.006

Vagostello, L., Oliveira, A. S., Silva, A. M., Donofrio, V., \& Moreno, T. C. M. (2003). Violência doméstica e escola: Um estudo em escolas públicas de São Paulo. Paidéia (Ribeirão Preto), 13(26), 191-196. doi:10.1590/S0103-863X2003000300008 
World Health Organization, \& International Society for Prevention of Child Abuse and Neglect. (2006). Preventing child maltreatment: A guide to taking action and generating evidence. Geneva, Switzerland: WHO.

Ana Carolina de Almeida Patrian é Psicóloga pela Universidade Federal de São Carlos.

Karyne de Souza Augusto Rios é Doutora em Educação Especial pela Universidade Federal de São Carlos.

Lúcia Cavalcanti de Albuquerque Williams é Professora Titular do Departamento de Psicologia da Universidade Federal de São Carlos.

Recebido: 20/03/2012

$1^{a}$ revisão: $21 / 08 / 2012$

$2^{a}$ revisão: $16 / 10 / 2012$

Aceite final: 28/11/2012

Como citar este artigo:

Patrian, A. C. A., Rios, K. S. A., Williams, L. C. A. (2013). Validade de critério do Inventário de Potencial para Abuso Infantil (CAP). Paidéia (Ribeirão Preto), 23(54), 43-51. doi:http://dx.doi.org/10.1590/1982-43272354201306 


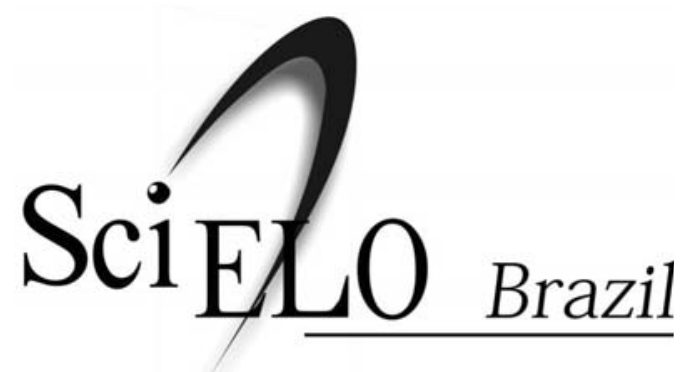
A Revista Paideia é uma publicação quadrimensal disponível integralmente na Biblioteca Científica Eletrônica Online - SciELO.

A SciELO permite o acesso fácil às tabelas de conteúdo e aos textos completos dos artigos.

\begin{abstract}
A SciELO permite também a recuperação de artigos por autor, palavras do título, resumo e descritores, além de fornecer indicadores bibliométricos e de consultas.
\end{abstract}

Os textos são enriquecidos com conexões para as bases de dados LILACS e MEDLINE.

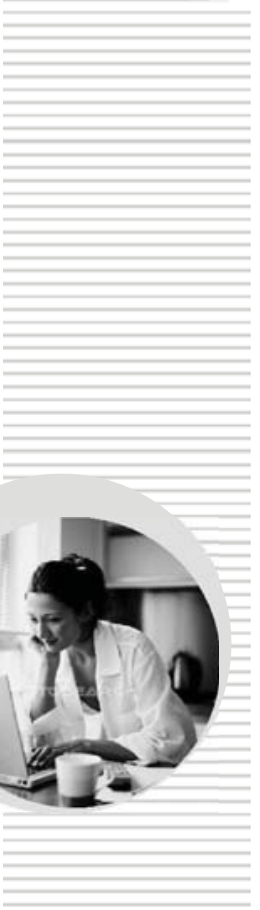

A Revista Paideia é parte integrante da SciELO Brasil:

\title{
www.scielo.br/paideia
}

O Portal SciELO integra e promove acesso rede de sites SciELO, reunindo as principais revistas científicas da América Latina e Espanha:

\section{www.scielo.org}

SciELO é fruto da cooperação entre FAPESP, a BIREME e editores científicos. 